Grand Valley State University

ScholarWorks@GVSU

$6-2002$

\title{
Blending the Roles of Home Care Nursing and Cardiovascular Specialties: A Model for Nursing Practice in the Community
}

Margaret A. Guthaus

Grand Valley State University

Diane White

Follow this and additional works at: https://scholarworks.gvsu.edu/kcon_articles

Part of the Nursing Commons

\section{ScholarWorks Citation}

Guthaus, Margaret A. and White, Diane, "Blending the Roles of Home Care Nursing and Cardiovascular Specialties: A Model for Nursing Practice in the Community" (2002). Peer Reviewed Articles. 18. https://scholarworks.gvsu.edu/kcon_articles/18

This Article is brought to you for free and open access by the Kirkhof College of Nursing at ScholarWorks@GVSU. It has been accepted for inclusion in Peer Reviewed Articles by an authorized administrator of ScholarWorks@GVSU.

For more information, please contact scholarworks@gvsu.edu. 


\title{
Blending the Roles of Home Care Nursing and Cardiovascular Specialties: A Model for Nursing Practice in the Community
}

\author{
Margaret A. Guthaus, MSN, RN, CS \\ Diane White, MSN, RN
}

\begin{abstract}
Changes in reimbursement and advances in technology have resulted in a decreased hospital stay for patients undergoing cardiac surgery. These patients are being discharged into the community setting earlier, resulting in an increased demand for transitional specialty home care. This article relates the experience of a Midwest home care agency charged with the development of specialty home care for these complex patients. It offers a new practice model for professional nursing staff delivering this level of care. This model challenges traditional beliefs that cardiac specialty home care can best be provided by nurses with critical care or acute care cardiology background. This agency was successfully able to use the specialty roles of generalist home care as a foundation for advanced cardiovascular practice in the home care setting due to the agency's commitment to support this process with an on-site advanced practice nurse skilled in both cardiovascular nursing and home health care.
\end{abstract}

$\mathrm{A}$ s the complexities and demands of cardiac home health care have escalated, so has the perception that nurses with critical care skills are best able to meet the postoperative needs of these patients (Lazarre \& Ax, 1995). This article will challenge these traditional beliefs and introduce a model for delivery of postdischarge cardiac surgical care that blends the specialty of home care nurse generalist prac- tice with the specialty of cardiovascular disease management and recovery.

\section{NEEDS OF THE POSTCARDIAC SURGICAL PATIENT}

In 1998, over three quarters of a million cardiac surgical procedures were performed in the United States. This reflects a $384 \%$ increase in the number of cardiac surgeries performed since 1979 (American Heart Association, 2000). During this same time period, the average length of hospitalization for cardiac surgery decreased from the traditional 7-to-12-day range to 5 days postsurgery (Frantz \& Walters, 2001; Leske \& Pelczynski, 1999). Within these brief hospitalizations, patients are often discharged with inadequate preparation to meet their complex health care needs and postoperative care requirements (Driscoll, 2000). This leads to a situation in which patients and families feel anxiety, stress, and inadequacy to fulfill their treatment plan for the early recovery period (Leske \& Pelczynski, 1999). Frequently added to these are issues with pain control, activity limitations, and sleep disturbances (Goodman, 1997), and general concerns about physical recovery and physiological adaptation (Moore, 1997). Ultimately, the early period can escalate to one in which the outcomes for patients and their families are adversely affected.

Key Words: home care, generalist, cardiac surgery, cardiovascular specialty

Home Health Care Management \& Practice / June 2002 / Volume 14, Number 4, 255-261 (C)2002 Sage Publications 
Home health nurses can facilitate a seamless transition to home by providing patient and family education and support that is difficult to provide during brief hospitalizations. Through informational support, families are better prepared to provide comfort and assistance during their loved one's postcardiac surgical recovery (Leske \& Pelczynski, 1999). The following describes a cardiovascular specialty program (CVSP) developed to meet these needs. The discussion includes the evolution of the nurse staffing mix, the supportive services required, the measurable outcomes, and recommendations for other programs.

\section{EARLY DEVELOPMENT OF THE CVSP}

A Midwest home care agency affiliated with a large tertiary care cardiovascular (CV) center was charged with developing a postoperative cardiac surgical program. This program was to address the complex recovery needs of recently discharged cardiac surgical patients and their families. In addition to responding to patient needs, it was equally imperative to consider community, physician, and payer concerns. Crucial to this process was the need to facilitate a "transition of trust" among all stakeholders across the continuum of care. To accomplish this, the home care agency identified the need to integrate home care standards with knowledge of advanced CV disease management principles, acute and home care pathways, and physician practice patterns.

Initially, the primary goal of the CVSP was to deliver consistent, collaborative care by a team of specialty nurses with advanced CV knowledge and expertise in evidence-based interventions. The specialty team of nurses was a hybrid of two subspecialties. The agency recruited nurses with experience in critical care (as recommended by Lazarre \& Ax, 1995) and with extensive experience in home care and medical surgical nursing and an interest in developing a specialized CV knowledge. This strategy reflected the agency's philosophy that home care in itself is a specialty practice.

The blending of both experienced CV nurses and home care nurses into a specialized team appeared to be an ideal approach. Several obvious benefits developed. Nurses were instrumental in the development of the CVSP from an integration of the two perspectives. Furthermore, recruiting from within the home care agency resources demonstrated fiscal accountability and contributed to the cost-effectiveness of the program. Adding the support and coordination of an advanced practice nurse (APN) with a clinical specialty in $\mathrm{CV}$ nursing further supported the blended role. The APN developed an educational program specifically for the provision of specialized CV home care that includes standardized, evidence-based interventions for optimum patient outcomes. The course was given prior to beginning practice. Once completed, each nurse was assigned to a geographical team as a CV specialty nurse.

The early program was successful in terms of improved patient outcomes and decreased readmission to acute care facilities. In addition, the program was effective in increasing the referral base, which supported new registered nurse positions within the $\mathrm{CV}$ team. Unfortunately, 18 months after the program was implemented, all of the critical care nurses hired specifically for the CVSP had resigned from home care practice. According to information obtained during exit interviews, dissatisfaction with the role was a major reason for their resignation decisions. In particular, these nurses expressed discomfort with the isolation and unpredictability of home care nursing practice. After an abbreviated home care experience, many nurses chose to return to the acute/critical care practice arena.

In addition to this information, the agency examined data related to referral sources and patient outcomes for the CVSP. Within 6 months, referrals to agency for patients with $\mathrm{CV}$ disease processes doubled. Cardiac surgeons associated with the tertiary medical center became the number one practice for agency referrals among all groups of physicians. Patients managed by blended-role CV staff demonstrated more expedient goal attainment and cost-effective use of agency and community resources. These outcomes included optimum recovery and return to role within the family and community. Patients experienced fewer complications such as wound infections and pleural effusion requiring intervention. This ultimately decreased emergent physician and acute care visits and readmission. Review of this data led to the agency's determination that of the two specialties, the home care generalist with CV knowledge was more adept at delivering nursing care in an unpredictable home environment. Furthermore, these nurses were highly motivated to expand their knowledge base in order to participate in this challenging and dynamic arena.

At this 18-month point, the agency was forced to respond to a number of other issues, including changing reimbursement systems, allocating scarce resources, 
expanding geographical responsibility, and serving an increasing CV census. These factors precipitated the shift to a new practice paradigm in which the generalist home health nurse would be prepared to manage complex CV patients at the same level as a specialty $\mathrm{CV}$ nurse. These nurses would assume the blended-practice role. CV patients would no longer be assigned to a $\mathrm{CV}$ team specialty nurse but managed by the home care generalist nurse assigned to that geographical area who had successfully completed the CVSP course.

The selection of nurses for the blended-practice role was the next challenge. Immediate supervisors recommended generalist staff who met stringent criteria developed collaboratively by the CV APN and the clinical management team. All nurses considered for this role were care managers who delivered home health in a specific geographic area. Their overall performance demonstrated effective and timely coordination of outcome-focused care, which was delivered within the agency's standard for productivity. In addition, the applicant's performance evaluations reflected a commitment to professional growth, development and utilization of critical thinking skills, and ownership of practice. For a comprehensive list of criteria used in this process, see Table 1.

A component of this criteria process was to identify competencies developed by today's successful generalist home health nurses. There are several major competencies that the home health generalist must demonstrate in order to respond to this level of service delivery. Changes in the acuity of the patient population due to decreased hospital length of stay have required increased accountability in the standards of home health care. Nurses are now caring for patients with more complex medical and technological needs and are providing care in the home for patients much earlier in their disease process (Ellenbecker \& Warren, 1998; Moulton, McGrand, Beck, Holland, \& Christopher, 1998). As the expectations and level of care have advanced, so has the need for autonomous critical thinking and anticipatory problem solving with patient and family dynamics. The integration of these skills in response to increased technology and acuity has resulted in care responsive to patient needs as they transition to home from the acute care environment.

Competent, interdisciplinary care management for cost-effective use of payer and community resources is essential. This includes precise screening and coordination of services that meet the challenges of reimbursement and regulation. Interdisciplinary team coordina-
TABLE 1

Employment and Staff Performance Criteria for Blended-Role Nurses

Criteria

Date of hire with agency

Length of time/quality of performance in care-management role

Application of nursing process

Individualization of care

Appropriate referrals and use of agency and community resources

Ability to coordinate complex care with multiple disciplines and providers

Mean productivity standard during previous 12 months

Timely clinical care path utilization within framework of reimbursement system

Summary overall ratings of evaluations

Corrective actions, informal counseling

Reliability, accuracy of regulatory documentation, and quality improvement participation

Effective interpersonal and care continuum communication Clinical judgment and critical thinking skills

Accountability and ownership of practice and professional development

tion is key to care management function in home care. The generalist who functions as a care manager facilitates team communication, strengthens collaboration, promotes a team direction, and assists the patient and family caregivers to set and meet mutually agreed-upon goals. A demonstrated level of nursing knowledge and skill, self-confidence, and advocating for patient services makes for solid partnerships, not only with the physician but also with clinicians in the varied health care environments.

Communication skills must reflect the ability of the nurse to relate effectively to diverse patient populations and all levels of care providers and their gatekeepers across the continuum. In the acute care or primary setting, the nurse may have direct access to the prescribing physician, whereas the home care nurse many times communicates through a third party. To avoid inappropriate interventions or changes in treatment plan, communication must be articulate and present a complete and accurate picture that is delivered with the appropriate sense of urgency. The expertise of this level of communication can be pivotal to the outcome of any patient receiving care in the current home health climate. 


\section{Summary}

All of these competencies and areas of expertise provide support for why the experienced home care nurse is the ideal professional to facilitate a patient's and family caregiver's transition from a high technological hospital to the residential environment. It is recognized that these roles and competencies are not unique to the home care nurse but are expectations of all professional nurses. However, a home care nurse's practice arena is in the home, which may present an element of variability and unpredictability that may be absent or lessened in other health care settings. Therefore, it can be concluded that this expertise of service delivery in the home setting would provide an effective foundation for specialty practice in the community.

\section{REQUIRED AGENCY SUPPORT FOR BLENDED ROLE}

Even though the blended role was perceived as advantageous, there was an apprehension that further integration and blending of the $\mathrm{CV}$ specialty would dilute the program. Therefore, the challenge was to maintain the same CVSP effectiveness and outcomes as when a CV specialty nurse delivered the service. There was also concern among the generalist staff who valued the diversity of their role. They were afraid that once they had cross-trained to CVSP, they would be expected to constantly shift their priorities to those patients. Despite these initial reservations, the benefits of implementing a blended-role practice model greatly outweighed any concerns. As the program was implemented, the agency support components required for the new delivery model was clearer. They included the advanced practice $\mathrm{CV}$ nurse, a specialty training module, and the opportunity for clinical validation as the newly learned knowledge was applied in the field.

\section{Role of Advanced Practice Nursing in Program Integration}

The integration of the generalist and CV specialty roles is an ideal opportunity for the APN in home care. The availability of a cardiology-based APN to develop and coordinate the process is pivotal to prepare the home care generalist to assume responsibility for cardiac surgical patients during their transition to home and self-care. This position is ideally suited to the educational preparation and functional roles of the $\mathrm{CV}$ clinical nurse specialist (CNS). Program development, educational presentations, and staff mentoring are necessary for clinical expert role credibility. Knowledge of research through both utilization and participation is required to maintain evidence-based interventions for current standards of care. The value of the APN in a home care agency is now well recognized and can be the difference between success and failure in blending these roles for specialty service delivery (Cyr, 1990; Lazarre \& Ax, 1995; Miller, 1995; Milone-Nuzzo \& Pike, 2001; Twardon \& Gartner, 1992).

\section{Educational Program and Clinical Validation}

The CV CNS had developed an educational program during the initial implementation of the CVSP. However, the background of the generalist nurse's transitioning into this role was varied and may not have included any acute cardiology care. Therefore, to provide the cardiovascular background necessary for these nurses to practice with the acuity of these patients in the home, the education program had to be completely restructured to include knowledge of interventions across the entire disease management continuum.

Basic electrocardiogram (ECG) rhythm interpretation was a required skill, as these nurses would be using ECG rhythms as a tool of assessment. Nurses were offered the option of attending a 15-hour class or acquiring this knowledge through a self-paced learning module. Following this certification, nurses began 14 hours of didactic instruction by the CV CNS, which included cardiac functional anatomy, pathophysiology, CV drugs, and advanced CV assessment. Disease management of various cardiac pathologies, including treatment with medical, interventional, or surgical procedures, was also presented. This lecture content was based on standards developed by the American Heart Association, American College of Cardiology, and the Agency for Health Care Policy and Research. Nursing interventions incorporated these standards and were presented within the framework of the Cardiovascular Nursing Practice and Home Health Practice by the American Nurses Association.

The course included a pretest and a comprehensive posttest that focused on application of knowledge learned specific to practicing $\mathrm{CV}$ nursing in the home care arena. In addition to the posttest, nurses completed motor competencies for correct use of ECG and pulse oximetry equipment. See Table 2 for a complete list of course content and competencies. 
TABLE 2

Cardiovascular (CV) Home Care

Course Content and Competencies

Content/Competencies

Basic electrocardiogram certification

Functional cardiac anatomy, physiology, and pathophysiology

Cardiovascular drugs_-vasodilators (central, alpha, peripheral, angiotension converting enzyme inhibitors, angiotension blocking agents), calcium channel and beta blockers, diuretics, nitrates, antiarrhythmics, and inotropes Management of pathology of coronary circulation (medical, interventional, and surgical)

Management of heart failure

Advanced CV assessment including history, physical exam, and interpretation of diagnostic data

An important component of the $\mathrm{CV}$ program was the development of clinical pathways for specific diagnoses. Again, these pathways were developed from evidenced-based intervention within the framework of nursing practice. The cardiac surgical home care pathway included assessment and education for recovery and long-term management of disease process. Interventions were outcome focused for patient goal attainment, and nonachievement was varianced and scored. The clinical paths were formatted to prompt the nurse to assess and educate in an orderly and consistent manner and yet allowed for individualization. The agency's clinical path process was not unique to the cardiac surgical patient but included all major disease processes managed by their home care staff. They included pathways for diabetes, chronic obstructive pulmonary disease, congestive heart failure, hypertension, pneumonia, ostomy, wound, and dementia. Table 3 is an example of a component of the cardiac surgical pathway that directed self-monitoring interventions for optimum recovery. This type of format is reflective of the agency's approach to providing clinical paths for optimum resource utilization and outcomes.

Once the nurse had completed the course, competencies, and use of clinical pathways, the CNS began a process of clinical validation and mentoring. Joint visits were made with all new staff to model CV service delivery and competencies. Once the nurse demonstrated ability to consistently provide the necessary level of care, independent visits were made; however, the CNS was accessible within the organization for ongoing consultation and direction to all staff delivering CVSP. This was probably the most crucial component of the change in the CVSP practice model. Validation of clinical judgment and problem solving by a CV expert was paramount to the home care nurse's successful development in this new role.

\section{MEASURABLE OUTCOMES OF PROGRAM}

Several benefits were noted from the implementation of a new practice model within the CVSP. The agency was able to quickly respond to the increased need for nursing services to care for the higher volume of $\mathrm{CV}$ patients in a cost-effective manner. By preparing home care generalists to provide specialized CV care in an expanded role, the nurses experienced improved morale though job security, satisfaction, and cohesiveness. As such, nurse turnover was minimized, and the agency was able to retain valued home care staff.

Furthermore, this blended model provided greater flexibility in scheduling and coordination of CVSP services. Through their blended roles, these nurses were able to provide an instant buffer as patient census fluctuated. Equally important was the ability of a single nurse to provide both $\mathrm{CV}$ and generalist care in rural areas. This blended role demonstrated optimal agency utilization of current resources given the change in home care reimbursement.

\section{Logistics of Implementation}

Agencies that provide home care to $\mathrm{CV}$ patients in their community may want to consider this practice model. The process begins with identifying the potential census and referral sources to evaluate whether CV specialty care is warranted and beneficial, not only for the community but also the viability of the agency. For this blended role to be successful, the agency must make the commitment to provide an onsite APN to develop, manage, and update program and staff. CNSs are educated in program development and mentoring clinical excellence. The ability to evaluate a specialty program and practice model implementation, with a systems perspective, will allow for integration that results in minimal disruption or significant change in agency process.

Implementation of this model did require additional equipment, as there were more nurses providing the cardiac home care. For this agency, the increased refer- 
TABLE 3

Education for Patient Self-Monitoring and Recovery

Topic
1. Daily weight
2. Temperature 2 times per day, call if higher than 101
3. Cough, deep breath, use of incentive spirometer
4. Incision care
5. Avoid caffeine
6. Signs and symptoms of atrial fibrillation
7. Signs and symptoms of incision complication
8. Strategies for sleep
9. Activity limitations
10. Signs and symptoms of fluid retention
11. Bowel management
12. Differentiating chest pain
13. Medication administration
14. Specific medication instruction
15. Sexual activity

Date Completed

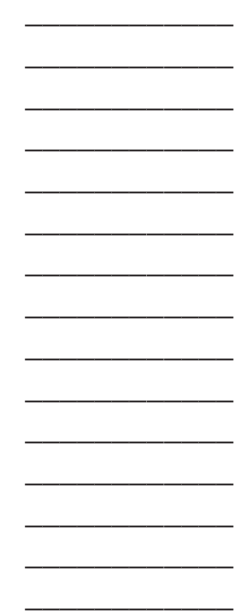

ral base, ability to respond to needs of integrated partners, and decreased travel of a team CV specialty nurse offset the additional expense.

Clinical pathways are essential for this model as they provide a guideline for standardized, evidence-based care. Combining the pathways with educational development of these blended nurses facilitates the delivery of consistent, outcome-focused care. Outcomes, both patient and system, must be regularly measured and used for improvement in program and service delivery. These outcomes can be measured from patient pathway indicators and agency and referral reports.

\section{RECOMMENDATIONS FOR HOME CARE AND NURSING}

It is time for home care agencies that service CV patients to consider developing a $\mathrm{CV}$ program incorporating advanced practitioner knowledge and evidencebased interventions to meet the transition needs of these patients. However, who and how best to deliver this level of service in the home setting remains open for debate. The complexities of delivering advanced education and technology in the home care setting require autonomy, integrated critical thinking, and the ability to provide interventions that foster patient and family caregiver self-management. Critical to the implementation of this agency's model was the commitment to provide an on-site CV CNS who was able to develop edu- cational programs, apply research and standards of care, and mentor the clinical role of these blended nurses. The clinician who assumes this role must not only be an expert in cardiology but also in the delivery of this level of care in the home setting. Additionally, he or she must foster collaborative relationships with referral sources and integrated partners. Without this level of resource, it would be difficult to fully integrate any specialty program into a generalist system.

Through patient outcomes, staff response, improved scheduling, and referral data, the new model has been effective in maintaining the standards of the CVSP. Job satisfaction and retention of those nurses functioning in the blended role is well established, and the need to educate other nurses continues to grow. All staff transitioned from generalist to blended role have remained within the agency

This model presents the opportunity for research that would directly measure patient and fiscal outcomes related to home care nursing competencies and education for integrated specialty practice. This type of research could provide support for utilization of a blended-role model for delivery of any specialty program in the home care arena. Given the current health care and reimbursement climate and the changing composition of home care patients, the tradition of utilizing critical/acute cardiology-experienced nurses for CV home care may be challenging due to cost constraints and a shortage of available nurses from acute care. This 
model offers another option for staying competitive by offering a CV specialty service while maximizing the use of valued and qualified home care generalists.

\section{REFERENCES}

American Heart Association. (2000). 2001 heart and stroke statistical update. Dallas, TX: Author.

Cyr, L. B. (1990). The clinical nurse specialist in a home healthcare setting. Home Heathcare Nurse, 8(1), 34-39.

Driscoll, A. (2000). Managing post-discharge care at home: An analysis of patients' and their caregivers' perceptions of information received during their stay in hospital. Journal of Advanced Nursing, 31, 1165-1173.

Ellenbecker, C. H., \& Warren, K. (1998). Nursing practice and patient care in a changing home healthcare environment. Home Healthcare Nurse, 16, 531-539.

Frantz, A., \& Walters, J. I. (2001). Cardiac home care programs impact patients after coronary artery bypass grafting. Home Healthcare Nurse, 19, 495-501.

Goodman, H. (1997). Patients' perceptions of their education needs in the first six weeks following discharge after cardiac surgery. Journal of Advanced Nursing, 25, 1241-1251.

Lazarre, M., \& Ax, S. (1995). Cardiac specialty program for home health care: A model for implementation. Journal of Home Health Care Practice, $8(1), 1-8$.

Leske, J. S., \& Pelczynski, S. A. (1999). Caregiver satisfaction with preparation for discharge in a decreased-length-of-stay cardiac surgery program. Journal of Cardiovascular Nursing, 14, 35-43.
Miller, S. E. (1995). The role of the clinical nurse specialist in home health care. Journal of Home Health Care Practice, 7, 62-72.

Milone-Nuzzo, P., \& Pike, A. (2001). Advanced practice nurses in home care: Is there a role? Home Health Care Management \& Practice, 13, 349-355.

Moore, S. (1997). Effects of interventions to promote recovery in coronary artery bypass surgical patients. Journal of Cardiovascular Nursing, 12, 59-70.

Moulton, P. J., McGrand, A. M., Beck, T. L., Holland, N. L., \& Christopher, M. A. (1998). Utilization of home health care services by elderly patients with heart failure. Home Health Care Management \& Practice, 10(4), 66-73.

Twardon, C., \& Gartner, M. (1992). A strategy for growth in home care: The clinical nurse specialist. Journal of Nursing Administration, 22(10), 49-53.

Margaret A. Guthaus, MSN, RN, CS, is the cardiovascular clinical nurse specialist at Borgess Visiting Nurse \& Hospice in Kalamazoo, Michigan. She has extensive experience in both medical and surgical cardiovascular care, and for the past 6 years she has practiced in the home care arena. She is responsible for the development and management of the agency's cardiovascular specialty program and also serves as adjunct faculty at Grand Valley State University.

Diane White, MSN, RN, is vice president of clinical services at Borgess Visiting Nurse \& Hospice in Kalamazoo, Michigan. She has an extensive background in community health nursing at both the academic and administrative levels. In addition to home care, she has administrative experience in pediatrics and maternal child in the acute and ambulatory care setting. 